\title{
Digital Video Recording in Traumatic Brain Injury Surgery Using a Wearable Action Camera
}

\author{
Eui Hyun Hwang, Tea Hoon Rho, Se-Hyuk Kim, Nam Kyu You \\ Department of Neurosurgery, Ajou University School of Medicine, Ajou University Hospital, Suwon, Republic of Korea
}

Corresponding author: Nam Kyu You

Department of Neurosurgery,

Ajou University School of Medicine, Ajou University Hospital, 164, World Cup-ro, Yeongtong-gu, Suwon 16499, Republic of Korea

Tel: +82-31-219-5230

Fax: +82-31-219-5238

E-mail: nkyou@ajou.ac.kr

Received: September 3, 2018 Revised: September 14, 2018 Accepted: September 17, 2018

\begin{abstract}
Objective: Video recording is essential to training and education in the medical field. The recorded videos are used to education. There is less chance of experience in emergent or urgent traumatic brain injury (TBI) surgeries for residents. We used wearable action camcorders for surgical image recording in TBI, then evaluated its safety and feasibility for education. Methods: A wearable action camera was used to record surgical procedures. High-definition $(1920 \times 1080 \mathrm{p}$ resolution, 60 frames per sec) quality was set and the camera was attached to the surgeon's head and recorded the view of the surgical field as seen by the surgeon. Burr hole trephination, craniotomy for hematoma removal, and decompressive craniectomy surgeries were recorded. The recorded video clips were stored securely and reviewed. To evaluate the usefulness of the images, we conducted reviews and surveys of the residents. Results: From January 2015 to December 2016, 81 cases of TBI surgery were recorded. Most video clips had a favorable view and image quality. Image distortion by overexposure occurred in 5 cases. Shut down due to misuse of device occurred in 2 cases and the recordings were out of focus in 5 cases. Results of the survey showed that TBI surgical video clips were helpful for understanding the sequence of surgery and sharing the field of view of the main operator. Conclusion: Wearable action camera can be used to record surgical video clips, including TBI surgery, and its videos may be helpful for the training and education of neurosurgery residents.
\end{abstract}

Key Words: Brain injuries, traumatic; Education; Neurosurgical procedures; Video recording

\section{INTRODUCTION}

Imaging recording in the surgical field is important for the education and training of new surgeons. In past decades, independent camcorders were used for recording, separated from the surgeon and another person was needed to record the images. Sometimes, a light-mounted recorder was used but it was expensive, and field of view was be limited by the position and motion of the surgeon. Some surgeons use commercially available digital camcorders and attach it on a handle of a surgical light or a holding arm for camera ${ }^{1,2)}$. A surgical microscope could record images but in the neurotrauma field, a microscope is used less frequently than other neurosurgical fields.

Recently, digital devices have become small enough to attach to a surgeon's head, and indeed recorded image quality has been improved. Wearable camcorders were introduced in the market for sports and leisure, but their performance and small size has also made them available in medical fields ${ }^{8,9)}$. We used one of these wearable and cheap devices for surgical image recording in traumatic brain injury (TBI), then evaluated its safety and feasibility.

\section{MATERIALS AND METHODS}

\section{Technical Specifications of the Camcorder}

We purchased an HX-A500 action camera (up to 32 GB storage -SDHC card, $2 \mathrm{hr}$ long internal battery, waterproof, Wi-Fi control, 1080 p 60 frames per sec [fps], 2160 p 24 fps; Panasonic, Osaka, Japan), which was cheaper than a professional video recording system.

\section{Wearing the Device}

A single surgeon used this camcorder as an operator during TBI surgery. This camera consisted of a small round camera unit and a square-shaped control unit connected to a $70-\mathrm{cm}$ long cable. The head mounting accessory was included and it was attached to the surgeon's head (Fig. 1). The control unit was attached to an armband on the surgeon's upper arm. It was controlled by push buttons on a surgical gown or by wire- 
Table 1. Survey for usefulness of action camcorder recording traumatic brain injury after watching selected video clips

\begin{tabular}{|c|c|}
\hline Questions & Answers (points) \\
\hline 1. Does the video image provide the whole sequence of surgery? & Never helpful (1) \\
\hline 2. Can you see from an operator's view, which provides a more detailed view of the operation field? & Seldom/rarely heplful (2) \\
\hline 3. Is the video image helpful for your surgery as a main operator? & Neutral (3) \\
\hline 4. Can you handle sudden change or emergency during operation after viewing the recording? & Usually helpful (4) \\
\hline $\begin{array}{l}\text { 5. Is the video image helpful for understanding the following steps? } \\
\text { (Scalp incision/Craniotomy/Dura incision/Removal of hematoma/Dura closure/Skull fixation/Wound closure) }\end{array}$ & Strongly helpful (5) \\
\hline
\end{tabular}

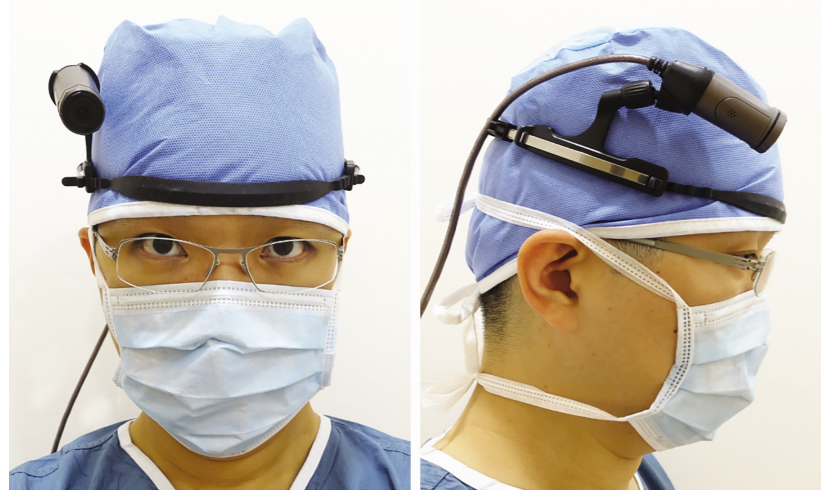

Fig. 1. Head-mounted action camcorder on the surgeon's head. Field of view was set at 50 to $60 \mathrm{~cm}$ anterior to eyes in the neutral position.

less remote control. The head unit and the arm control unit were worn before surgical scrubbing.

\section{Recording and Storage of Video Files}

Recording image quality was set to 1080 p 60 fps with normal field of view. Movie files were stored with h264 codec, in 0.19 GB size per min. Starting time was decided by the surgeon and scalp incision and retraction was not routinely recorded due to limited battery life.

Recorded video files were transferred immediately after surgery in a password-locked hard disk drive on a Windows computer system. They were sorted by date and reviewed. Video files were reviewed by a neurosurgeon. We checked the following aspects: the center of the image matched to the surgeon's view or center of surgical field, image quality, and overexposure or underexposure which can distort the image detail.

\section{Review by Residents}

Recorded movie clips were shown to the residents of our institute. First-grade residents were not included as reviewers because they had not experienced an operating room. A total of six residents (from $2^{\text {nd }}$ to $4^{\text {th }}$ grade, each grade having two people) participated in reviewing the images and then answered the questions (Table 1) We assessed the understanding of the
Table 2. Characteristics of recorded cases

\begin{tabular}{lc}
\hline \hline & $N(\%)(\mathrm{n}=81)$ \\
\hline Mex & \\
Female & $67(82.7)$ \\
Age & $14(17.3)$ \\
Craniotomy & $49.0 \pm 20.2$ years \\
Craniectomy & $26(32.1)$ \\
Cranioplasty & $32(39.5)$ \\
Burr hole/Shunt & $9(11.1)$ \\
\hline
\end{tabular}

The data is presented as number (\%) or mean \pm standard deviation.

whole sequence of surgery, sharing of operator's field of view, education for the main operator, and how to manage an emergency situation. According to the degree of assistance provided by the video recording, 1 to 5 points could be assigned to each of the assigned criteria.

\section{RESULTS}

\section{Characteristics of Recorded Cases}

From January 2015 to December 2016, 81 surgical cases were recorded. Craniotomy and craniectomy cases were 58 and trephination cases were 14 (Table 2). The mean recording time was $48 \mathrm{~min}$. Detachment of the camcorder did not occur during the operations. The total size of recorded files was $731.9 \mathrm{~GB}$ and average data ratio was $4.2 \mathrm{~GB}$ per $22 \mathrm{~min}$. The field of view was larger than the main operation field in most cases. However, in some cases focus was displaced from the center, especially in the early period of use.

\section{Image Quality}

The captured video images were well identifiable. Decompressive craniectomy was easily comprehensible step by step (Fig. 2). Frontal craniotomy and frontal sinus exposure in frontal sinus fracture in patients with cerebrospinal fluid leakage were recorded (Fig. 3). After craniectomy and durotomy, swelling of the brain was recorded, including severe brain swelling, known 

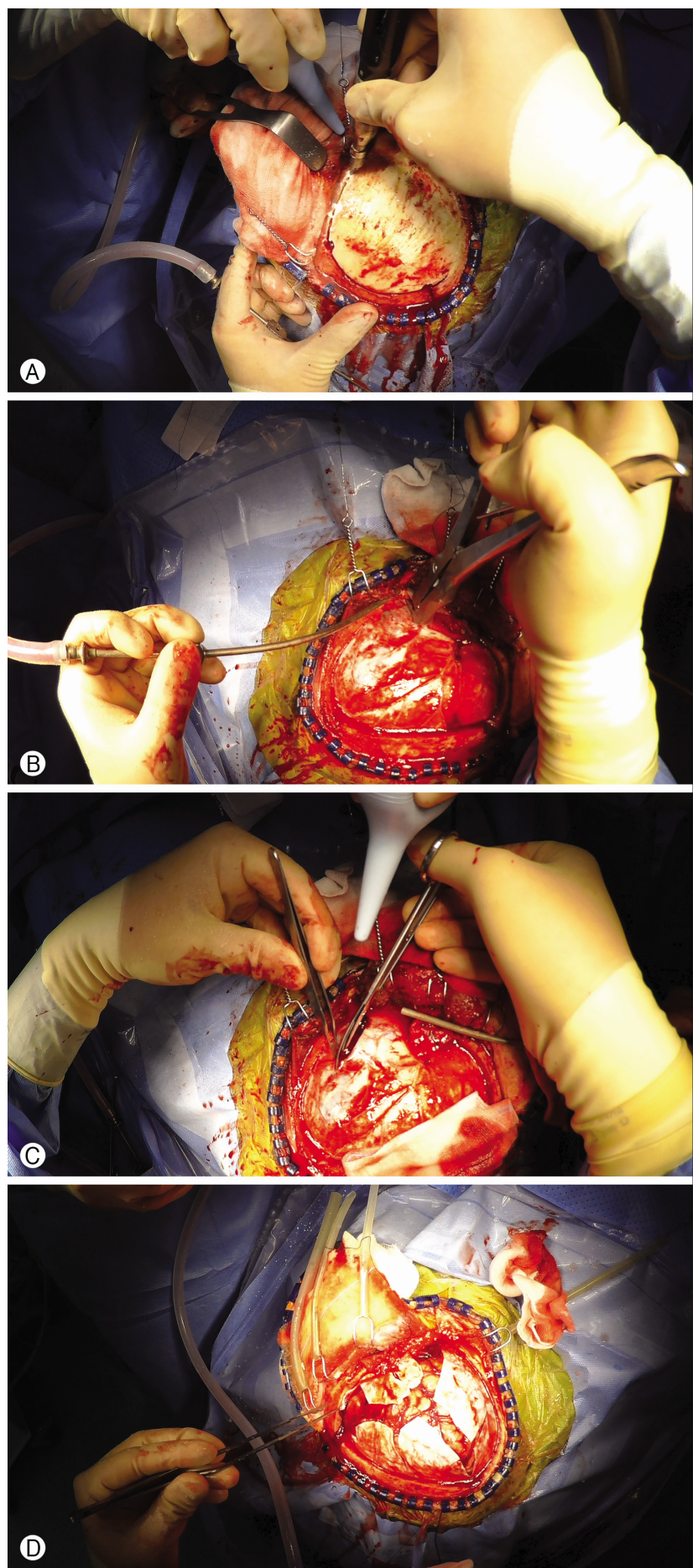

Fig. 2. (A) Craniotomy for subdural hematoma using an electric highspeed drill. (B) Temporal bone resection with a rongeur for decompression. (C) Dura incision. (D) After decompression brain swelling which caused widening of the dura matter, then cerebral cortex was exposed between dura opening.

as angry brain (Fig. 4). There were three angry brain cases. Exposure of image was controlled by the camera automatically.

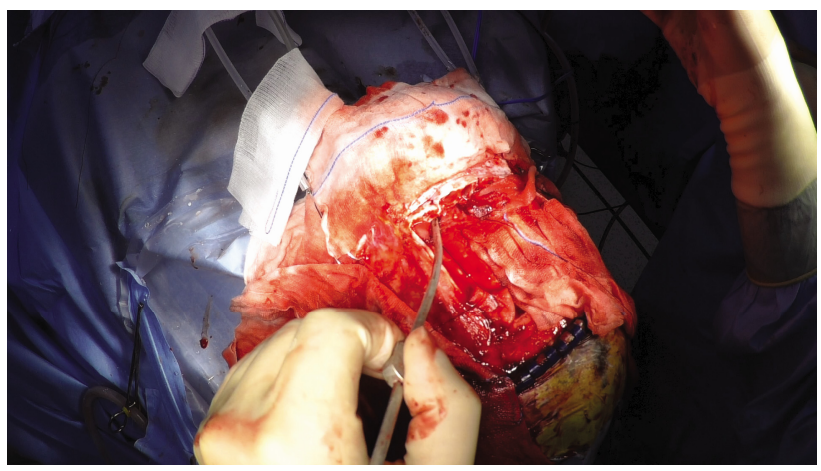

Fig. 3. Bifrontal craniotomy and exposure of frontal sinus and dura tearing area.

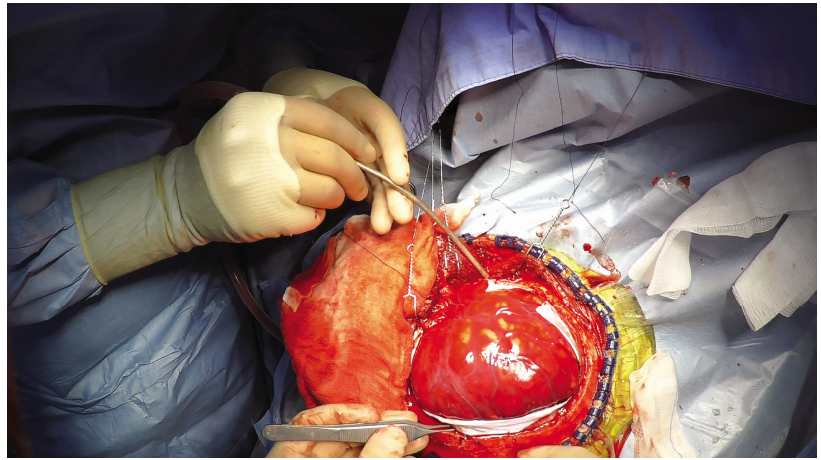

Fig. 4. Diffuse brain swelling after dura incision. Red colored cerebral cortex with severe swelling called angry brain.

Overexposure caused image distortion and it happened in 5 cases. The high intensity of surgical light caused the distortion of color and detail in highlighted parts of the video. Recording time was enough to contain the most important sequence of surgery.

\section{Complication and Malfunction of the Recorder}

The camcorder never detached during the operations. It did not interrupt the view and movement of the assistant operator. There was no case of surgery-related infection. Unwanted camcorder shut down occurred in 2 cases and it was due to consumption of the battery. Unintended and unpredicted ceasing of recording occurred in 2 cases, because of the pressure on the buttons of the control unit during the operation.

\section{Review by Residents}

We selected four surgical recording movie clips. Removal of epidural hematoma, removal of subdural hematoma, decompressive craniectomy, and cranioplasty in patients with cerebrospinal fluid leakage. The 6 residents submitted their survey after reviewing the movie clips. The overall degree of help was rated at 4.26 of 5 points (Table 3). Understanding 
Table 3. Results of the survey

\begin{tabular}{|c|c|c|c|c|c|c|c|}
\hline \multirow{2}{*}{$\begin{array}{l}\text { Questions } \\
\text { 1. Does the video image provide whole sequence of surgery? }\end{array}$} & \multicolumn{2}{|c|}{ R4 } & \multicolumn{2}{|c|}{ R3 } & \multicolumn{2}{|c|}{ R2 } & \multirow{2}{*}{$\frac{\text { Mean }}{4.00}$} \\
\hline & 5 & 4 & 4 & 4 & 4 & 3 & \\
\hline 2. Can you see from an operator's view, which provides a more detailed view of the operation field? & 4 & 5 & 5 & 4 & 4 & 4 & 4.33 \\
\hline 3. Is the video image helpful for your surgery as a main operator? & 5 & 5 & 4 & 4 & 5 & 4 & 4.50 \\
\hline 4. Can you handle sudden change or emergency during operation after viewing the recording? & 4 & 4 & 4 & 3 & 3 & 4 & 3.67 \\
\hline \multicolumn{8}{|l|}{ 5. Is the video image helpful for understanding the following steps? } \\
\hline Scalp incision & 4 & 5 & 4 & 4 & 5 & 4 & 4.33 \\
\hline Craniotomy & 5 & 4 & 5 & 4 & 5 & 4 & 4.50 \\
\hline Dura incision & 4 & 5 & 4 & 5 & 3 & 4 & 4.17 \\
\hline Removal of hematoma & 5 & 4 & 4 & 5 & 4 & 3 & 4.50 \\
\hline Dura closure & 4 & 4 & 5 & 5 & 4 & 5 & 4.17 \\
\hline Skull fixation & 4 & 4 & 5 & 4 & 4 & 4 & 4.50 \\
\hline Wound closure & 4 & 4 & 5 & 5 & 4 & 5 & 4.26 \\
\hline
\end{tabular}

R4: $4^{\text {th }}$ grade resident; R3: $3^{\text {rd }}$ grade resident; R2: $2^{\text {nd }}$ grade resident.

whole sequence of surgery, sharing operator's field of view, and help for future main operators showed higher points. However, a relatively low point was seen in the handling of emergent situations.

\section{DISCUSSION}

In our study with the wearable action camera in TBI surgery recording, surgical video clips were easily obtained and had good image quality for replay and capture for still images. The battery life was less than $2 \mathrm{hr}$, but after scalp retraction, the main period of surgery was recorded in most cases. After adaption of the head mount position, out of center was rarely identified in the review.

\section{Properties of Wearable Action Camcorder Necessary for Recording during Surgeny}

Simple control, such as start and stop recording was possible by pushing the button on the device, but real-time Wi-Fi transmission of image needed another device, such as a tablet personal computer or smartphone. This part of the procedure may need covering of aseptic pouch or another person to control it. Real-time image may be used for live surgery. Some devices also have voice command functions ${ }^{3,6,9)}$.

Battery life is sufficient for recording the essential parts of surgery, but it cannot record the full length of the operation from opening to closure of the incision. If there are consecutive surgeries, not enough recording time is available without changing the battery. Since the wearable camcorders are not expensive, a second device during recording or a device with a rechargeable battery can be obtained.

Nakhla et al. ${ }^{10)}$ reported on the usefulness of Google Glass (Google Inc, Mountain View, CA, USA) in surgical education of neurosurgery residents. We used a camera and body separated model. As most other devices are one-body structure, and head-mounting may cause headache or neck pain in the surgeon. An eyeglass-type recording device may reduce these disadvantages, but the image quality of current models is low and have shorter battery time $e^{6,9,10)}$.

Surgical lighting is important for the recording image quality due to the performance of the action camera. High-intensity light may cause overexposure in most digital recording devices. We experienced some overexposed cases in the early period of recording. Before recording, it is better to lower the intensity of light for better image quality.

The technology needs to be improved to make future devices of smaller size and lighter weight, with higher image quality. They should also be able to record for longer times with extended battery life. High-quality image transmission via a wireless network, as well as real-time interaction, should be possible from the device itself.

\section{Education with Surgical Video Clips}

Surgery videos, recorded in this manner, may help to train neurosurgery residents and contribute to the further development of a surgeon himself. Safety of the patients and prognosis will be improved. In the past years, surgical recording needed manpower, expensive equipment, and preparation before surgery. In most TBI surgeries done in emergent or urgent situations, participating chance may not be equal in every trainee. High-quality video clips will help every trainee to learn about the surgery. We assessed the usefulness of surgical video clips in TBI surgery by surveying the residents. Residents had the experience of a main operator, and were aware of dynamic changes during surgery, and how to handle to it. Practical experience provides better understanding than textbooks and visualization of the experience of established medical profe- 
ssionals assists trainees in getting acquainted with their medical fields. The power of video recording in medical fields has been reported and suggested ${ }^{7}$. Microscopic or laparoscopic surgeries have incorporated video clips in most cases and they were helpful for resident or junior surgeon training ${ }^{4,5)}$. TBI surgery is done as an emergent surgery in most cases. Since it is difficult for new trainees to directly perform such crucial surgeries without experience, these video recordings contribute to their learning process

\section{CONCLUSION}

We presented digital image recording in the field of neurotrauma surgery using the wearable camcorder system. It produced relatively high-quality images which provided important data for clinical practice, teaching, and preparing presentations. It is necessary to improve these small digital recording devices and use more widely in the surgical field.

\section{CONFLICT OF INTEREST}

No potential conflict of interest relevant to this article was reported.

\section{REFERENCES}

1. Chen CM, Lee KT, Shen YH: Simple method to record highquality surgical videos. Br J Oral Maxillofac Surg 47:494, 2009
2. Coronel-Banda ME, Serra-Renom JM, Lorente M, Larrea-Terán WP: Mucosal perforasomes. Plast Reconstr Surg 132:1081e1082e, 2013

3. Ehrler F, Siebert J, Haddad K, Sahin A, Schrurs P, Diener R, et al.: Adapting guidelines for google glass: the case of pediatric CPR. Stud Health Technol Inform 224:141-145, 2016

4. Frykman PK, Duel BP, Gangi A, Williams JA, Berci G, Freedman AL: Evaluation of a video telescopic operating microscope (VITOM) for pediatric surgery and urology: a preliminary report. J Laparoendosc Adv Surg Tech A 23:639-643, 2013

5. Herrera-Almario GE, Kirk K, Guerrero VT, Jeong K, Kim S, Hamad GG: The effect of video review of resident laparoscopic surgical skills measured by self- and external assessment. Am J Surg 211:315-320, 2016

6. Lee CK, Kim Y, Lee N, Kim B, Kim D, Yi S: Feasibility study of utilization of action camera, GoPro hero 4, google glass, and panasonic HX-A100 in spine surgery. Spine (Phila Pa 1976) 42: 275-280, 2017

7. Makary MA: The power of video recording: taking quality to the next level. JAMA 309:1591-1592, 2013

8. Matsumoto S, Sekine K, Yamazaki M, Funabiki T, Orita T, Shimizu M, et al.: Digital video recording in trauma surgery using commercially available equipment. Scand J Trauma Resusc Emerg Med 21:27, 2013

9. Muensterer OJ, Lacher M, Zoeller C, Bronstein M, Kübler J: Google Glass in pediatric surgery: an exploratory study. Int J Surg 12:281-289, 2014

10. Nakhla J, Kobets A, De la Garza Ramos R, Haranhalli N, Gelfand Y, Ammar A, et al.: Use of google glass to enhance surgical education of neurosurgery residents: "proof-of-concept" study. World Neurosurg 98:711-714, 2017 